\title{
A simple test of muscle coactivation estimation using electromyography
}

U.F. Ervilha, T. Graven-Nielsen and M. Duarte

The Brazilian Journal of Medical and Biological Research is partially financed by

\section{惡CNPq} Conselho Nacional de Des
Cientifico e Tecrológico
Ministério da Ciência e Tecnologia

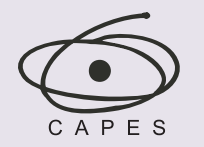

Ministério da Educação

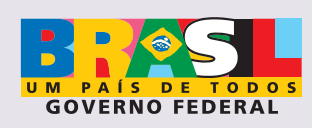

\section{Institutional Sponsors}

suifo
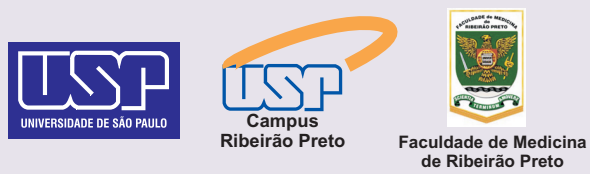

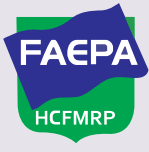

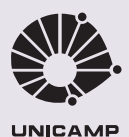

UNICAMP

\section{Ф SHIMADZU}

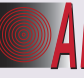

AfFl

Associa
Fundo

Fundo
de Incentivo à Pesquisa

Explore High - Performance MS Orbitrap Technology

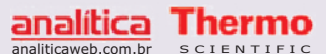




\title{
A simple test of muscle coactivation estimation using electromyography
}

\author{
U.F. Ervilha1 ${ }^{1}$ T. Graven-Nielsen ${ }^{2}$ and M. Duarte ${ }^{3}$ \\ ${ }^{1}$ Laboratório de Biodinâmica do Movimento Humano, Escola de Educação Física, \\ Universidade São Judas Tadeu, São Paulo, SP, Brasil \\ ${ }^{2}$ Center for Sensory-Motor Interaction, Aalborg University, Aalborg, Denmark \\ ${ }^{3}$ Programa de Engenharia Biomédica, Universidade Federal do ABC, Santo André, SP, Brasil
}

\begin{abstract}
In numerous motor tasks, muscles around a joint act coactively to generate opposite torques. A variety of indexes based on electromyography signals have been presented in the literature to quantify muscle coactivation. However, it is not known how to estimate it reliably using such indexes. The goal of this study was to test the reliability of the estimation of muscle coactivation using electromyography. Isometric coactivation was obtained at various muscle activation levels. For this task, any coactivation measurement/index should present the maximal score (100\% of coactivation). Two coactivation indexes were applied. In the first, the antagonistic muscle activity (the lower electromyographic signal between two muscles that generate opposite joint torques) is divided by the mean between the agonistic and antagonistic muscle activations. In the second, the ratio between antagonistic and agonistic muscle activation is calculated. Moreover, we computed these indexes considering different electromyographic amplitude normalization procedures. It was found that the first algorithm, with all signals normalized by their respective maximal voluntary coactivation, generates the index closest to the true value (100\%), reaching $92 \pm 6 \%$. In contrast, the coactivation index value was $82 \pm 12 \%$ when the second algorithm was applied and the electromyographic signal was not normalized $(P<0.04)$. The new finding of the present study is that muscle coactivation is more reliably estimated if the EMG signals are normalized by their respective maximal voluntary contraction obtained during maximal coactivation prior to dividing the antagonistic muscle activity by the mean between the agonistic and antagonistic muscle activations.
\end{abstract}

Key words: Muscle coactivation; Electromyogram; Muscle cocontraction; EMG index

\section{Introduction}

Muscle coactivation, the simultaneous activation of agonist and antagonist muscle groups around a joint $(1,2)$, is an important and common strategy for the control of voluntary movement in humans and has been experimentally observed during a wide variety of conditions including locomotion (3), isometric and functional activities (4), low and high accurate pointing tasks $(2,5)$, upright standing (6), central nervous system impairment $(7,8)$, and lumbopelvic stabilization (9), among others. In the control of movements, it has been suggested that muscle coactivation modulates the impedance of a joint, mainly stabilizing the joint (2).

In experimental conditions, coactivation is most often estimated by comparing the amplitude of the myoelectric activity of muscles that generate opposite torques during a task. Despite the fact that a muscle can be active more than another and yet generate less force (or vice versa) due to a number of factors, including pennation angle and fiber length, and also considering that caution should be used if the relationship between force and electromyographic (EMG) signal is not established for all the muscles investigated, various indexes of coactivation based solely on the EMG signal have been proposed (10-17). However, it is not known how coactivation based on EMG is reliably estimated with such indexes. The goal of this study was to test the reliability of the estimation of muscle coactivation using EMG.

\section{Subjects and Methods}

\section{Subjects}

Ten volunteers with no history of neuromuscular disorders ( 6 men and 4 women; mean \pm SD: age $=25 \pm 5$ years, height $=176 \pm 10 \mathrm{~cm}$, and mass $=74 \pm 11 \mathrm{~kg}$ ) participated in the experiment. The study was conducted in accordance

Correspondence: U.F. Ervilha, Laboratório de Biodinâmica do Movimento Humano, Escola de Educação Física, Universidade São Judas Tadeu, Rua Taquari, 546, 03166-000 São Paulo, SP, Brasil. E-mail: prof.ulysses@saojudas.br

Received August 15, 2011. Accepted May 17, 2012. Available online June 1, 2012. Published September 3, 2012. 
with the Helsinki Declaration and was approved by the Aalborg University Ethics Committee (VN2003/61). The volunteers received information about the experiment and gave written informed consent to participate.

\section{Indexes of coactivation}

Two indexes were selected to represent most of the coactivation indexes $(\mathrm{Cl})$ based on the amplitude of the EMG signal employed in the literature (10-17). In the first index $\left(\mathrm{Cl}_{1}\right)$, the antagonistic muscle activity (the lower EMG amplitude between two muscles that generate opposite joint torques) is divided by the mean between the agonistic $\left(E M G_{A G}\right)$ and antagonistic $\left(E M G_{A N T}\right)$ muscle activations (10). The second index $\left(\mathrm{Cl}_{2}\right)$ is obtained by calculating the ratio between antagonistic and agonistic muscle activation (11). The formulas for these indexes are shown below:

$$
C I_{1}=2 * \frac{E M G_{A N T}}{E M G_{A G}+E M G_{A N T}} * 100(1) \quad C I_{2}=\frac{E M G_{A N T}}{E M G_{A G}} * 100
$$

The method for EMG amplitude normalization used when coactivation is calculated varies in the literature. The EMG signals have often been normalized by the maximum voluntary isometric contraction (MVIC) $(13,18)$, the maximum voluntary isometric coactivation value (MVICa) (10), or have not been normalized $(19,20)$. Here we will investigate how these three methods affect the $\mathrm{Cl}$.

\section{Protocol}

Coactivation around a joint that does not move and with no external moments implies that the net joint moment is equal to zero. Such task can be performed at any level of muscle activation, as long as the joint does not move (full coactivation). Thus, the volunteers were instructed to activate simultaneously elbow extensor and flexor muscles in order to achieve full coactivation at various levels of muscle activation. In order to allow the volunteers to maintain a targeted level of muscle activation, established for that task, the EMG linear envelope from the medial belly of the biceps brachii muscle was shown in real time on an oscilloscope. The volunteers practiced the task prior to data collection by performing 3 to 4 times $2 \mathrm{~s}$ of full coactivation at various muscle activation levels. The data for 1 volunteer was not included in the study because she was unable to perform the task. The subjects rested for 1 min between two practicing trials. A full coactivation was then carried out for 4 $\mathrm{s}$, at different biceps muscle activation levels $(25,50$, and $75 \%$ ) of the biceps muscle EMG activity achieved during a maximal effort muscle activation (100\%) keeping a full coactivation. At the beginning of the session, the volunteers performed a coactivation at a maximum effort so the EMG activity from the medial head of the biceps brachii muscle could be used as the reference for the biofeedback and the peak EMG from the biceps and triceps brachii muscles could be used for EMG normalization. For a second EMG normalization procedure, the peak EMG from a maximal isometric voluntary contraction for elbow flexors and elbow extensors was used.

\section{Setup}

The volunteers were comfortably seated with the arm supported at $90^{\circ}$ abduction and $90^{\circ}$ elbow flexion. A pair of surface electrodes (Medicotest 72001-k, Denmark) was placed in the direction of the muscle fibers (2-cm apart) on shaved, cleaned skin. On the biceps brachii muscle, the electrodes were placed on the medial and lateral head, on the lead-line between the acromion and the fossa cubit at $1 / 3$ from the fossa cubit. On the triceps brachii muscle, the electrodes were placed on the lateral and medial head - 1 $\mathrm{cm}$ lateral to the lead-line just on the midpoint between the acromion and the olecranon process. The EMG signals were bandpass filtered (2nd order, 20 to $500 \mathrm{~Hz}$ ), amplified 1000 times (CounterPoint MK2, Dantec, Denmark) and sampled at $1024 \mathrm{~Hz}$.

\section{Data analysis and statistics}

The digital EMG signal was band-pass filtered (2nd order, zero-phase-lag Butterworth, 20 to $400 \mathrm{~Hz}$ ), full wave rectified, and smoothed (low-pass, 4th order, zero-phase-lag Butterworth filter with a $3-\mathrm{Hz}$ cutoff frequency). From the $4 \mathrm{~s}$ of coactivation, a 1-s interval was extracted in which the squared difference between the acquired EMG and the targeted EMG intensity was the lowest. This procedure allowed us to select the 1-s window where the subject's EMG activation was closest to the target displayed on the oscilloscope. The difference between the target versus the actual EMG activation level was calculated as percent error.

Data are reported as means $\pm S D$. Three-way repeated analyses of variance (ANOVA) were used to examine the effects of $\mathrm{Cl}\left(\mathrm{Cl}_{1}\right.$ and $\left.\mathrm{Cl}_{2}\right)$, normalization procedures [nonnormalization (NN), normalization by the MVIC (NMVIC), and normalization by the MVICa (NMVICa)], and muscle activation level $(25,50,75,100 \%)$ on the coactivation index. When ANOVA was found to be significant, the Student-Newman-Keuls post hoc test was used for multiple comparisons. The level of significance was set at $P<0.05$.

\section{Results}

Figure 1 shows representative plots of the EMG time series for different target levels and the respective error for one subject. The means \pm SD for the 10 subjects for the 25 , 50,75 , and $100 \%$ levels were $3 \pm 3,3 \pm 1,5 \pm 3$, and $10 \pm$ $6 \%$, respectively. These low values suggest very consistent patterns of activation if we consider that the subjects had as feedback the EMG values of their muscles, which are well known to be very variable. Based on the variability of the results, we suggest that the subjects were familiarized with the task and that they performed it as they were asked 

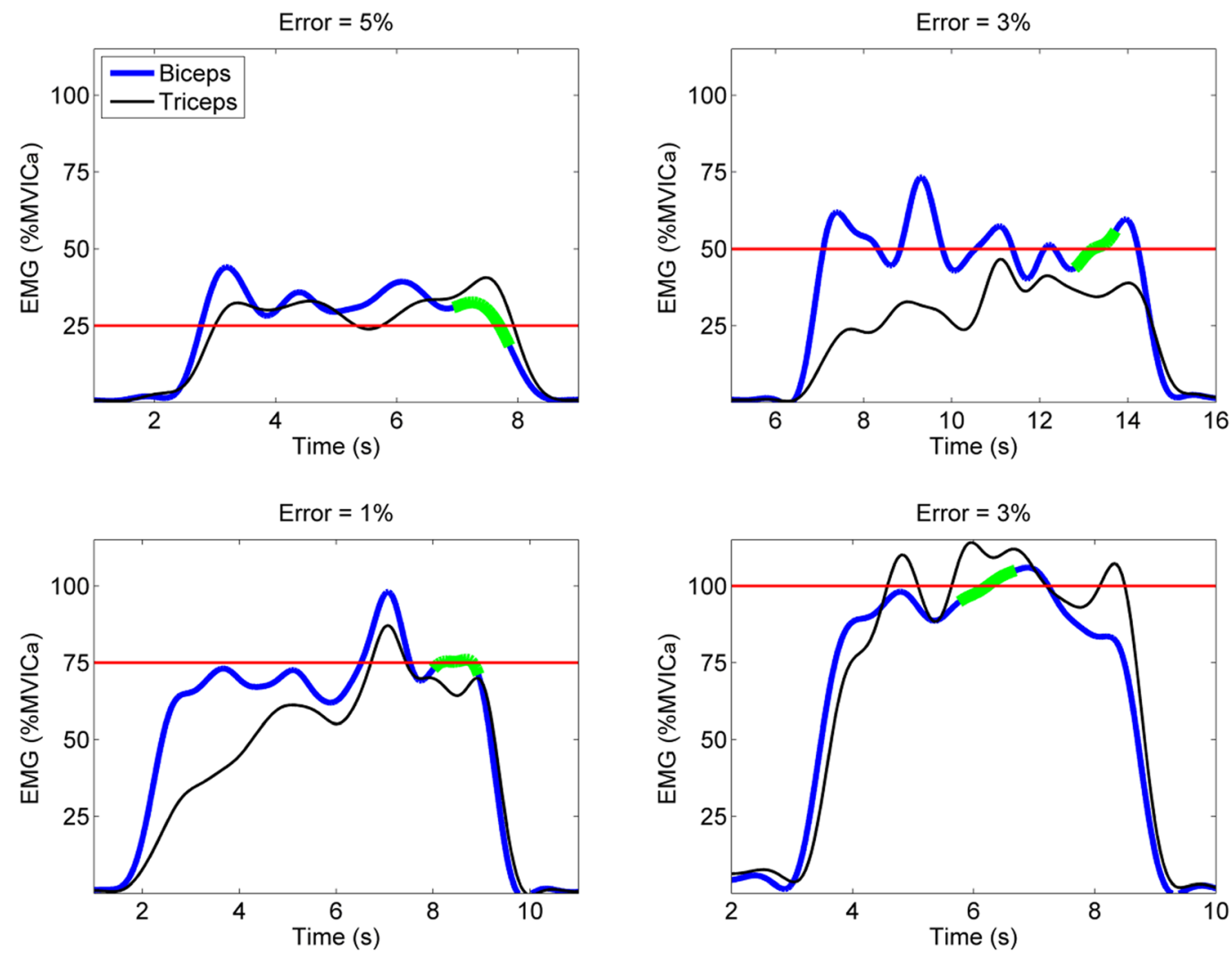

Figure 1. Determination of how far (\% error) the actual electromyographic (EMG) signal is from the desired one for different target levels $(25,50,75$, and $100 \%$ of muscle activation, horizontal line) for one subject during the task. The thicker line for biceps muscle EMG represents the 1-s selected interval. MVICa = maximal voluntary isometric coactivation.

to. Although the variability throughout the 4-s contractions was quite large, as shown in Figure 1, it is important to emphasize the fact that the volunteers were requested to maintain the contraction at the target for $2 \mathrm{~s}$ only in order to avoid fatigue (as instructed in the familiarization training). Moreover, the fact that a 1-s window was selected makes the behavior during the other $3 \mathrm{~s}$ less important for the present investigation.

The mean values and standard deviation of the two coactivation indexes considering the different normalization methods applied are shown in Figure 2.

ANOVA for the factors algorithm $\left(\mathrm{Cl}_{1}\right.$ and $\left.\mathrm{Cl}_{2}\right)$, normalization procedures (NN, NMVIC, NMVICa), and muscle activation levels $(25,50,75$, and $100 \% \mathrm{MVIC})$ revealed a main effect of algorithm $(F(1,9)=137, P<0.001)$ and $a$ main effect of normalization procedure $(F(2,18)=3.9, P=$ 0.04). The post hoc analyses revealed that the $\mathrm{Cl}$ calculated by applying Equation $1\left(\mathrm{Cl}_{1}\right)$ had a higher value than that calculated by applying Equation $2\left(\mathrm{Cl}_{2}\right)$ for all normalization procedures, with respective pooled means \pm SD values of $87 \pm 10$ and $78 \pm 15 \%(P<0.03)$. Moreover, the $\mathrm{Cl}$ was higher when the EMG signal was normalized by the NMVICa compared to the $\mathrm{Cl}$ obtained when the EMG was not normalized for both indexes (NMVICa > NN; $\mathrm{P}<0.04$ ); pooled means \pm SD respectively equal to $89 \pm 6$ and $77 \pm 12 \%$. No interactions among factors were found by ANOVA.

\section{Discussion}

In this study, we verified the accuracy of $\mathrm{Cl}$ based on EMG recordings. Our results showed that none of the investigated $\mathrm{Cl}$ was able to accurately estimate the level of coactivation, which was theoretically known. The present data shows that muscle coactivation is more reliably estimated if the EMG signals are normalized by their respective maximal voluntary contraction obtained during maximal coactivation, prior to dividing the antagonistic muscle activity by the mean between the agonistic and antagonistic muscle activations. The inability of surface EMG electrodes to accurately record all motor units equally, the fact that not all muscles involved are recorded, and data processing limitations certainly contributed to coactivation values 
different from the theoretical expected ones. Furthermore, several muscles act at a joint and it seems to be crucial to consider the contribution of all muscles involved, as well as possible nonlinearities to reliably calculate coactivation. Note, however, that these very same limitations are present in the great majority of studies that employ EMG signals to estimate muscle coactivation (e.g., 2, 10-12, 3, 13, 14, 7, $15,8,16,17,6,5,4,9)$. Our rationale is that the low accuracy of the $\mathrm{Cl}$ we found, which certainly resulted from the far from perfect methods employed here (and elsewhere), exposes the limitation of such $\mathrm{Cl}$.

When muscles that produce torque in opposite directions are simultaneously activated, they limit the net moment generated at that joint. If the resultant contraction is an active maintenance of a static joint positioning, the muscles surrounding the joint are in full coactivation at any point from the minimum to the maximum muscle activation. However, any estimation of coactivation at that point must show that the net torque around that joint is zero, which is represented by the highest score when coactivation is measured.

In the present study, there was no joint movement or resultant external torques, implying that the volunteers had their muscles around the elbow in full coactivation, meaning zero net torque. In that case, any elbow flexor muscle is an antagonist to an elbow extensor and vice versa. In order to have a reference, the higher and the lower EMG intensity levels were considered for agonistic and antagonistic muscle activation, respectively. In theory, a $\mathrm{Cl}$ at $100 \%$ would be expected in all conditions studied in the present investigation. It was shown that a $\mathrm{Cl}$ that accounts for the antagonist torque generated at the joint and also for the additional agonist torque required to compensate for it, as proposed in $\mathrm{Cl}_{1}$, is more reliable because the index value reaches values closer to the maximum score. Moreover, there was no significant difference in the $\mathrm{Cl}$ calculated for different EMG levels, indicating that neither $\mathrm{Cl}$ is affected by the muscle activation level.

In order to minimize inter-subject EMG differences, the EMG signal amplitude was normalized. Various EMG amplitude normalization methods have been used in the literature when a $\mathrm{Cl}$ is calculated $(10,12,16,17)$. In the present study, it was shown that the EMG normalization procedure also influences the $\mathrm{Cl}$ outcome. When the EMG amplitude was normalized by the peak EMG signal obtained during a maximal full coactivation, the $\mathrm{Cl}$ approached the expected values for that motor task better than when the EMG amplitude was not normalized. Possibly, this is because this

\section{References}

1. Levine MG, Kabat $\mathrm{H}$. Cocontraction and reciprocal innervation in voluntary movement in man. Science 1952; 116: 115-118.

2. Hogan N. Adaptive control of mechanical impedance by

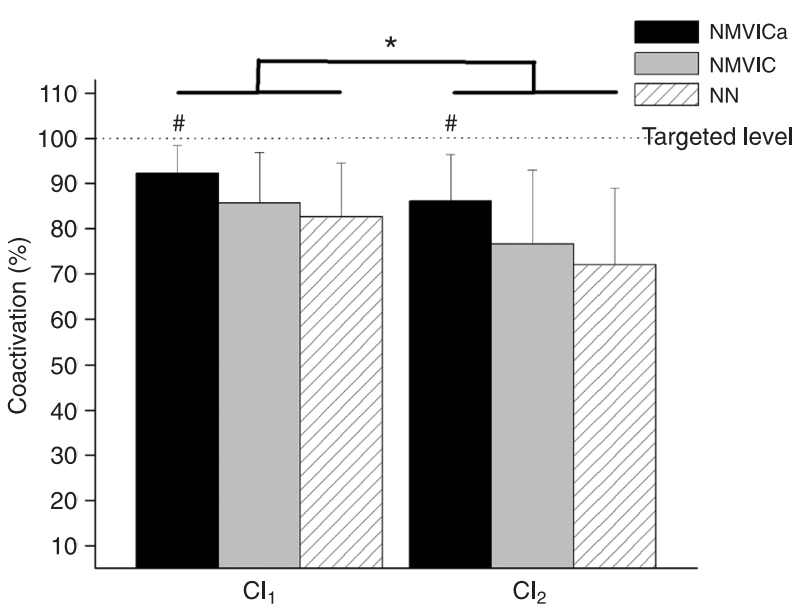

Figure 2. Coactivation indexes $\left(\mathrm{Cl}_{1}\right.$ and $\left.\mathrm{Cl}_{2}\right)$ based on electromyographic (EMG) signals (data pooled from 25, 50, 75, and $100 \%$ of muscle activation levels) non-normalized (NN), normalized by the maximal voluntary isometric contraction (NMVIC), or normalized by the maximal voluntary isometric coactivation (NMVICa). Data are reported as means $\pm \mathrm{SD}$. ${ }^{*} \mathrm{Cl}_{1}$ significantly higher than $\mathrm{Cl}_{2}$ for all normalization procedures $(\mathrm{P}<0.001)$. \#Coactivation index normalized by NMVICa significantly higher than the non-normalized coactivation index for both indexes $(P<0.04$; Student-Newman-Keuls test).

normalization procedure is related to the targeted task. That is, full coactivation at various muscle activation levels.

The present results were derived from a controlled task designed to validate the different methods to quantify coactivation. The task was always performed in a static condition and at the same position $\left(90^{\circ}\right.$ elbow flexion). Since muscle force is affected by its length and velocity (21) and during dynamic tasks the length and velocity of the muscles vary differently, these two factors might limit even more the use of $\mathrm{Cl}$ based on the EMG signals.

Furthermore, the low accuracy of the $\mathrm{Cl}$ suggests that coactivation indexes should be interpreted with caution and any methodological difference in the calculation should be considered before a comparison of different studies is performed.

\section{Acknowledgments}

M. Duarte was supported by a research grant from FAPESP (\#08/10461-07). 
4. Busse ME, Wiles CM, van Deursen RW. Co-activation: its association with weakness and specific neurological pathology. J Neuroeng Rehabil 2006; 3: 26.

5. Ervilha UF, Arendt-Nielsen L, Duarte M, Graven-Nielsen T. The effect of muscle pain on elbow flexion and coactivation tasks. Exp Brain Res 2004; 156: 174-182.

6. Benjuya N, Melzer I, Kaplanski J. Aging-induced shifts from a reliance on sensory input to muscle cocontraction during balanced standing. J Gerontol A Biol Sci Med Sci 2004; 59: 166-171.

7. Damiano DL, Martellotta TL, Sullivan DJ, Granata KP, Abe MF. Muscle force production and functional performance in spastic cerebral palsy: relationship of cocontraction. Arch Phys Med Rehabil 2000; 81: 895-900.

8. Chae J, Yang G, Park BK, Labatia I. Muscle weakness and cocontraction in upper limb hemiparesis: relationship to motor impairment and physical disability. Neurorehabil Neural Repair 2002; 16: 241-248.

9. Belavy DL, Richardson CA, Wilson SJ, Rittweger J, Felsenberg D. Superficial lumbopelvic muscle overactivity and decreased cocontraction after 8 weeks of bed rest. Spine 2007; 32: E23-E29.

10. Falconer K, Winter DA. Quantitative assessment of cocontraction at the ankle joint in walking. Electromyogr Clin Neurophysiol 1985; 25: 135-149.

11. Osternig LR, Hamill J, Lander JE, Robertson R. Co-activation of sprinter and distance runner muscles in isokinetic exercise. Med Sci Sports Exerc 1986; 18: 431-435.

12. Hammond MC, Fitts SS, Kraft GH, Nutter PB, Trotter MJ, Robinson LM. Co-contraction in the hemiparetic forearm: quantitative EMG evaluation. Arch Phys Med Rehabil 1988; 69: 348-351

13. Unnithan VB, Dowling JJ, Frost G, Volpe Ayub B, Bar-Or O.
Cocontraction and phasic activity during GAIT in children with cerebral palsy. Electromyogr Clin Neurophysiol 1996; 36: 487-494.

14. Frost G, Dowling J, Dyson K, Bar-Or O. Cocontraction in three age groups of children during treadmill locomotion. $J$ Electromyogr Kinesiol 1997; 7: 179-186.

15. Lamontagne A, Richards CL, Malouin F. Coactivation during gait as an adaptive behavior after stroke. J Electromyogr Kinesiol 2000; 10: 407-415.

16. Macaluso A, Nimmo MA, Foster JE, Cockburn M, McMillan NC, De Vito G. Contractile muscle volume and agonistantagonist coactivation account for differences in torque between young and older women. Muscle Nerve 2002; 25: 858-863.

17. Kellis E, Arabatzi F, Papadopoulos C. Muscle co-activation around the knee in drop jumping using the co-contraction index. J Electromyogr Kinesiol 2003; 13: 229-238.

18. Hurd WJ, Chmielewski TL, Snyder-Mackler L. Perturbationenhanced neuromuscular training alters muscle activity in female athletes. Knee Surg Sports Traumatol Arthrosc 2006; 14: 60-69.

19. Hopkins JT, Ingersoll CD, Sandrey MA, Bleggi SD. An electromyographic comparison of 4 closed chain exercises. $J$ Athl Train 1999; 34: 353-357.

20. Heise CO, Goncalves LR, Barbosa ER, Gherpelli JL. Botulinum toxin for treatment of cocontractions related to obstetrical brachial plexopathy. Arq Neuropsiquiatr 2005; 63: 588-591.

21. Heckman CJ, Weytjens JL, Loeb GE. Effect of velocity and mechanical history on the forces of motor units in the cat medial gastrocnemius muscle. J Neurophysiol 1992; 68: 1503-1515. 